\title{
Discovery and Characterization of Human Amniochorionic Membrane Microfractures
}

\author{
Lauren S. Richardson, ${ }^{*}$ Gracie Vargas, ${ }^{\dagger}$ Tyra Brown, ${ }^{\dagger}$ Lorenzo 0choa, ${ }^{\dagger}$ Samantha Sheller-Miller, ${ }^{*}$ George R. Saade, ${ }^{*}$ \\ Robert N. Taylor, ${ }^{\ddagger}$ and Ramkumar Menon*
}

\begin{abstract}
From the Division of Maternal-Fetal Medicine \& Perinatal Research,* Department of Obstetrics \& Gynecology, and the Department of Neuroscience \& Cell Biology and Center for Biomedical Engineering, ${ }^{\dagger}$ The University of Texas Medical Branch at Galveston, Galveston, Texas; and the Department of Obstetrics and Gynecology, ${ }^{\ddagger}$ Wake Forest School of Medicine, Winston-Salem, North Carolina
\end{abstract}

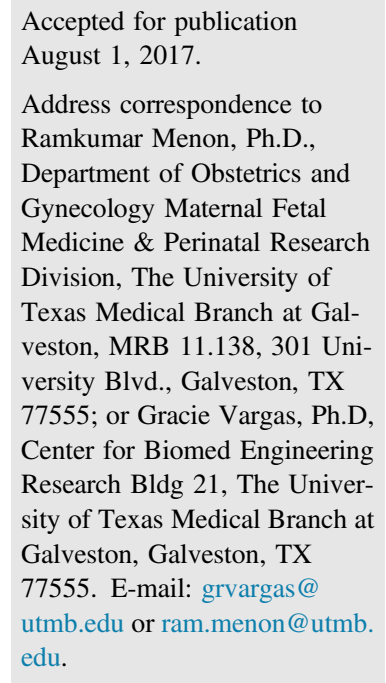

\begin{abstract}
This study obtained visual evidence of novel cellular and extracellular matrix-level structural alterations in term and preterm human fetal amniochorionic membranes. Amniochorions were collected from term cesarean (not in labor) or vaginal (labor) deliveries, preterm premature rupture of membranes, and spontaneous preterm birth. To determine the effect of oxidative stress on membranes at term or preterm labor, term not in labor samples in an organ explant culture in vitro were exposed to cigarette smoke extract. Tissues were imaged using multiphoton autofluorescence and second harmonic generation microscopy. Images were analyzed using ImageJ and IMARIS software. Three-dimensional microscopic analysis of membranes revealed microfractures that were characterized by amnion cell puckering, basement membrane degradation, and tunnels that extended into the collagen matrix with migrating cells. Numbers of microfractures were similar at term regardless of labor status; however, morphometric measures (width and depth) were higher in term labor membranes. 0xidative stress induced higher numbers of microfractures in term not in labor membranes, with morphometry resembling that seen in term labor membranes. Preterm premature rupture of the membranes had the highest number of microfractures compared to membranes from term and other preterm births. Microfractures are structural alterations indicative of areas of tissue remodeling during gestation. Their increase at preterm and in response to oxidative stress may indicate failure to reseal, predisposing membranes to rupture. (Am J Pathol 2017, 187: 2821-2830; https://doi.org/10.1016/j.ajpath.2017.08.019)
\end{abstract}

Fetal membranes (amniochorionic or placental membranes) comprise a vital intrauterine compartment, where they perform mechanical, immune, and endocrine functions to promote growth of the fetus and protection from environmental adversity. ${ }^{1}$ Membrane integrity is normally maintained throughout gestation, mediated by a well-balanced homeostasis involving matrix-degrading collagenolytic enzymes and their inhibitors. $^{2-4}$ Recently, we reported that amniochorionic membrane senescence occurs as a concomitant of fetal growth and tissue aging. ${ }^{5}$ Tissue damage arising from amniochorion senescence produces a sterile inflammatory response associated with parturition. ${ }^{6}$ Thus, amniochorionic membranes play major roles during gestation and parturition.

Amniochorionic membranes anatomically consist of a single layer of cuboidal amnion epithelial cells, chorionic trophoblasts, and scattered fibroblasts connected by a layer of type IV collagen-rich extracellular matrix. ${ }^{7}$ Basement membrane degradation by specific matrix degrading enzymes, as well as generalized proteolysis, is a factor predisposing to mechanical rupture of membranes before parturition at term or preterm. ${ }^{7} \mathrm{~A}$ reported feature of amniochorionic membrane rupture that provides evidence of these structural changes is the so-called zone of extreme altered morphology that overlies the site of rupture. $^{8}$ Morphometric characteristics of the zone of extreme altered morphology is also suggestive of membrane aging because cells and organelles are often enlarged and express

Supported by NIH/National Institute of Child Health and Human Development (NICHD) grant 1R01HD084532-01A1 (R.M.) and in part by a March of Dimes Ohio Center Innovative Catalyst grant and NICHD grant 1R03HD086354-01 (R.M.).

Disclosures: None declared. 
sterile inflammatory markers such as matrix metalloproteinase 9. Although these changes may be expected in membranes at term, similar features were seen in cases with early $(<34$ weeks $)$ preterm premature rupture of the membranes (pPROM) and spontaneous preterm birth.

Understanding the progressive morphological changes that occur in human amniochorionic membranes in utero is challenging because prospective, invasive sampling is unethical. Noninvasive imaging technologies to reliably scan amniochorionic membranes are yet to be developed. Even if such methodologies were discovered, structural alterations indicating imminent rupture or predicting preterm labor need to be established. We have embarked on the first step of this approach by applying sophisticated microscopic techniques to define structural changes in amniochorionic membranes in normal and pathologic pregnancies. By annotating amniochorionic membrane structural changes, we hope to provide insights into the mechanistic pathways contributing to such changes.

In the current studies, a combination of multiphoton autofluorescence microscopy and second harmonic generation microscopy was employed to generate three-dimensional multilayer representations of the surface organization of amniochorionic membranes. ${ }^{10}$ This approach, a combination of multiphoton autofluorescence and second harmonic generation microscopy, allows label-free, deep-tissue imaging of cellular and extracellular components of the amniochorionic membranes, through the collection of intrinsic autofluorescent signals. ${ }^{11-17}$ Using this approach, we were able to observe amnion epithelial cell shedding and gap formation at term, along with evidence of loosened collagen in the underlying extracellular matrix. ${ }^{17}$

The current study was undertaken to develop a microscopic method to allow more accurate volumetric characterization of amniochorionic membrane microstructural organization and to compare features between normal term births and pregnancies complicated by preterm delivery. Regions spanning the amniochorion and extracellular matrix extending all of the way to the maternal decidua were examined, and cellular morphology was determined and specifically examined for structural alterations. The discovery of unique microdomains-referred to as microfractures - in the amniochorionic membranes is reported. These microfractures are a structural feature in the fetal membrane and represent a break (fissure) in the continuity of the amnion surface that extends through the spongy layer and areas of sparse collagen, through to the reticular layer of the chorion basement membranes. We have categorized characteristic morphometric features of these microfractures and discuss their physiological and pathologic significance.

\section{Materials and Methods}

Amniochorionic membrane samples were obtained for this study from John Sealy Hospital at The University of Texas Medical Branch (UTMB) at Galveston, Texas, under an approved institutional review board protocol (UTMB 11-251).
Amniochorionic Membrane Collection for Multiphoton Microscopy Analysis

Gestational age-matched term amniochorionic membranes were used for this study. Amniochorionic membranes were collected from term not in labor cesarean deliveries, laboring vaginal deliveries after spontaneous onset of labor, and artificial rupture of the membranes (between 39 and 41 weeks) before delivery with no prior history of preterm birth or pPROM. Term membranes included in this study were limited to artificial rupture of the membranes because factors associated with spontaneous rupture of the membranes (at term) can be confounded by a variety of factors that could manifest in multiple structural outcomes. Collection, processing, and preparation of tissues for imaging are detailed in our prior publication. ${ }^{17}$ The methods used did not have any impact on collagen or other cellular features reported in this paper. Amniochorionic membranes from cesarean deliveries after pPROM, spontaneous preterm birth, or preeclampsia were also collected. Membranes were dissected from the placenta, washed three times in normal saline, and then cleaned of decidua and blood clots using cotton gauze. Biopsies $6 \mathrm{~mm}$ in diameter (explants) were then cut from the midzone of the reflected membranes, approximately 2.5 inches away from the cervix or placenta. Explants were then stored in $500 \mu \mathrm{L}$ of $10 \%$ formalin in 1.5-mL Eppendorf tubes until imaging.

For membranes from case groups from preterm births, the following factors were matched: gestational age, maternal age, and other sociodemographic variables (eg, race/ ethnicity, education, income, and marital and insurance status). Latency between labor (all subjects had a minimum rate of 2 contractions per 10 minutes) leading to delivery were matched in preterm birth and pPROM cases.

\section{Inclusion Criteria}

Women who had preterm birth were diagnosed with contractions (rate of 2 contractions per 10 minutes) leading to delivery with intact membranes or with pPROM (as determined by nitrazine test or amniotic fluid pooling) between $24^{0 / 7}$ weeks and $33^{6 / 7}$ weeks). Preeclampsia was defined in our study as blood pressure $\geq 140 / 90 \mathrm{~mm} \mathrm{Hg}$ on two occasions at least 4 hours apart after 20 weeks' gestation with new-onset proteinuria. Normal term birth was defined as term labor and delivery ( $>39^{0 / 7}$ weeks) with no pregnancyrelated complications.

\section{Exclusion Criteria}

Subjects with multiple gestations, placenta previa, fetal anomalies, and/or medical treatment or surgeries (interventions for clinical conditions that are not linked to pregnancy) during pregnancy were excluded. Severe cases of preeclampsia (persistent symptoms (headache, vision changes, right upper quadrant pain), abnormal laboratory findings (thrombocytopenia, repeated abnormal liver function tests, creatinine doubling or $>1.2$, or HELLP syndrome), or clinical findings 
(pulmonary edema or eclampsia) were excluded. Subjects who had any surgical procedures during pregnancy or who were treated for hypertension, preterm labor, or for suspected intraamniotic infection (eg, reports of foul-smelling vaginal discharge, high levels of c-reactive protein, fetal tachycardia) and delivered at term were excluded from the control groups.

\section{In Vitro Amniochorionic Membrane Organ Culture and Stimulation with Cigarette Smoke Extract}

The in vitro organ explant culture system for human amniochorionic membranes and stimulation of membranes with cigarette smoke extract were as previously reported. ${ }^{9}$ In this study, cigarette smoke extract was used to mimic the oxidative stress experienced by amniochorionic membranes at term before labor that transition the membrane into a labor phenotype. ${ }^{5}$ In short, 6-mm biopsies of amniochorionic membranes were collected from term not in labor cesarean deliveries and placed in an organ explant system for 24 hours. Cigarette smoke extract was prepared by bubbling smoke drawn from a single lit commercial cigarette (unfiltered Camel; R.J. Reynolds Tobacco Co., Winston Salem, NC) through $50 \mathrm{~mL}$ of tissue culture medium (Ham's F12/Dulbecco's modified Eagle's medium mixture with antimicrobial agents), which was then filter sterilized through a 0.22-mm filter (Millipore, Bedford, MA) to remove contaminant microbes and insoluble particles. Amniochorionic membranes were then stimulated with cigarette smoke extract (1:25 dilution) for 48 hours $(n=5)$, whereas the term not in labor control medium was replaced with tissue culture medium $(n=5)$. After a 48-hour treatment, the explants were removed and placed into $500 \mu \mathrm{L}$ of $10 \%$ formalin in 1.5-mL Eppendorf tubes for fixation before imaging.

\section{Optical Clearing}

Optical clearing of whole tissue explants to render the amniochorionic membranes optically transparent was performed by incubation of fixed amniochorionic membrane explants in 2,2'-thiodiethanol (refractive index 1.52), (SigmaAldrich St. Louis, MO). 2,2'-Thiodiethanol solutions (50\% and $100 \% \mathrm{w} / \mathrm{w}$ ) were prepared in Milli-Q water (Merck Millipore, Billerica, MA) at room temperature. Fixed tissue was completely immersed in 50\% 2,2'-thiodiethanol for 2 hours, and then in $100 \%$ 2,2'-thiodiethanol overnight at room temperature. Samples were then switched to fresh $100 \% 2,2^{\prime}-$ thiodiethanol for mounting and imaging as described above.

\section{Tissue Mounting}

For mounting, the tissue was placed with the amnion side facing a \#1.5- $\mu \mathrm{m}$ cover glass in a mounting chamber (30-mm cage plate; ThorLabs, Newton, NJ), with a second cover glass placed on the chorion side; $300 \mu \mathrm{L}$ of phosphate-buffered saline was added to the tissue, and the coverslips were tightened to secure the phosphate-buffered saline and tissue in the imaging chamber. The amnion epithelium was then oriented toward the objective on the upright microscope and centered with the help of an aiming beam from the excitation source.

\section{Imaging}

\section{Microscopy}

To determine the cellular and structural alterations in detail, a combination of multiphoton autofluorescence microscopy and second harmonic generation microscopy was used. This approach was used to investigate the multilayered threedimensional micro-organization of human amniochorionic membranes without the use of exogenous contrast agents, as previously applied by our group. ${ }^{17}$ Multiphoton autofluorescence microscopy and second harmonic generation microscopy were conducted using a Prairie Ultima IV upright microscope (Prairie Technologies/Bruker, Middleton, WI) using excitation from a Ti:sapphire femtosecond laser (Mai Tai; Spectra-Physics, Santa Clara, CA). A 25× objective with 1.05 numerical aperture (XLPlanN; Olympus, Tokyo, Japan) was used for image collection. Samples were illuminated at $840 \mathrm{~nm}$ for generation of both multiphoton autofluorescence microscopy and second harmonic generation microscopy, collected in an epiillumination geometry. Collected autofluorescence and second harmonic generation microscopy was split into two detection paths using a dichroic mirror, and autofluorescence emission was detected at $>500 \mathrm{~nm}$, whereas second harmonic generation microscopy collected at 420 $\mathrm{nm}$. Multiphoton autofluorescence microscopy and second harmonic generation microscopy were collected simultaneously in a 2-channel configuration using Gallium arsenide phosphide photomultiplier tubes (Hamamatsu Photonics, Hamamatsu, Japan) for detection.

\section{Image Collection}

Regions of interest were obtained ( 1 second per frame; $512 \times 512$ pixels or $1024 \times 1024$ pixels). Depth scans were obtained using a z-interval of $1 \mu \mathrm{m}$, with imaging depth ranging from 110 to $400 \mu \mathrm{m}$ depending on sample preparation as specified below. Images were obtained using a digital zoom factor of 1.19 , resulting in a field of view of $408 \times 408$ $\mu \mathrm{m}$. Image stacks were analyzed for epithelial characteristics, epithelial shedding and gaps, and collagen alterations using ImageJ software bundled with 64-bit Java version 1.8.0_112 (NIH, Bethesda, MD; http://imagej.nih.gov/ij), whereas IMARIS software version 7.6.5 (Bitplane, Concord, MA) was used for three-dimensional reconstructions of multiphoton autofluorescence microscopy and second harmonic generation microscopy stacks.

\section{Microfracture Quantification}

The total area of a 6-mm explant can be imaged by taking 25 to 30 individual images (mosaic tiling as reported earlier ${ }^{17}$ ) and stitching them together with ImageJ. The 

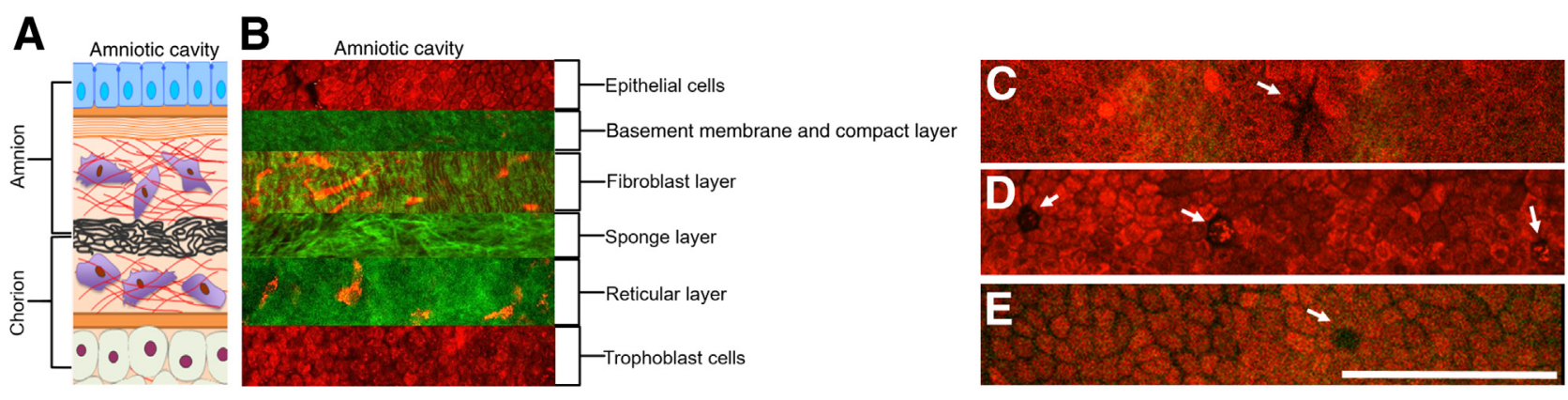

Figure 1 A and B: Characterization of amniochorionic membranes with nonlinear optical microscopy (A) compared to the layers imaged by a two-photon laser using multiphoton autofluorescence microscopy (red) and second harmonic generation microscopy (green) (B). A: A schematic of the amniochorionic membrane layers: Description starts from the innermost layer (amnion) facing the amniotic cavity and ends with the chorion. Amnion epithelial cells (blue) are connected to the first layer of the extracellular matrix, called the basement membrane (orange)/compact layer (orange strips). The fibroblast (top red), spongy (black), and reticular layers (bottom red) follow, containing stromal cells (purple). The chorion (tan) is connected to the extracellular matrix through a pseudobasement membrane (orange). B: Multiphoton autofluorescence microscopy and second harmonic generation microscopy of fetal membranes: Multiphoton autofluorescence microscopy (red) shows cuboidal amnion epithelial cells in a monolayer attached to the basement membrane in green as delineated by second harmonic generation microscopy. The layers of the extracellular matrix (green), including the basement membrane/compact layer and fibroblast, spongy, and reticular layers, exhibit unique collagen characteristics and contain stromal cells (red). Chorion trophoblast cells (red) are connected to the extracellular matrix. C: Amnion epithelial cells forming an area of altered morphology, puckering, as indicated by the white arrow. D: Amnion epithelial cells (white arrows) that are shedding away from the monolayer by losing junctions to neighboring cells. E: Amnion epithelial cell gap (white arrow), which leave holes in the epithelial layer. Scale bar $=150 \mu \mathrm{m}(\mathbf{C}-\mathbf{E})$. Images in B obtained with a $25 \times$ objective.

center nine images, captured with a $25 \times$ objective, were used as the normalizing surface area to serve as the common denominator for microfracture quantification. Microfractures in membranes were counted in a $1.50 \times 10^{6}-\mu \mathrm{m}^{3}$ area with ImageJ. This allowed us to normalize the surface area scanned for determining microfracture quantities. Microfractures were sliced and three-dimensionally reconstructed with IMARIS software to determine depth and width for each category studied. Kruskal-Wallis followed by Dunn's comparison between groups and $U$-test statistics were used to compare the clinical groups; significant differences were defined as $P<0.05$ in two-tailed tests.

\section{Results}

Amniochorionic Membrane Architecture as Determined by Multiphoton Autofluorescence Microscopy/Second Harmonic Generation Microscopy

The multilayered architecture of the human amniochorionic membranes were analyzed by reconstructed multiphoton autofluorescence microscopy and second harmonic generation microscopy methods as shown in Figure 1, A and B. Multiphoton autofluorescence microscopy was generated from endogenous cytoplasmic fluorophores and pseudocolored red in all images; fibrillar collagen autofluorescence in the extracellular matrix was captured by second harmonic generation microscopy and pseudocolored green (Figure 1B). Features associated with each layer are shown, with the signal arising from the amnion being due to cellular autofluorescence because no fibrillar collagen to produce second harmonic generation microscopy is found in that layer (thus, no green signal). Multiphoton autofluorescence microscopy allowed for visualization of cells in the fibroblast and reticular layers embedded within the extracellular matrix.
The amnion, stromal cells, and chorion cells were studied in the context of their extracellular matrix interfaces. As previously described, this method allows the identification of surface topography, collagen distribution, and cellular density within unique cell layers. ${ }^{17}$ Characterization of the amnion epithelial layer topography specifically revealed areas of surface disturbances (Figure 1C), epithelial shedding (Figure 1D), and epithelial gaps (voids lacking an epithelial cell, that extend through the single amnion layer, exposing the extracellular matrix) (Figure 1E).

\section{Discovery and Characterization of Microfractures}

Analysis of amnion topography identified areas within the epithelial monolayer that showed intercellular gaps (Figure 2, A and B). This is consistent with epithelial cell shedding. Morphological puckering (Figure 2C) or indentation of the monolayer surface was also noted. Analysis of orthogonally sliced sections showed areas of degraded basement membrane (Figure 2C) containing migrating epithelial cells (Figure 2D). These are shed or migratory cells that appear to invade degraded collagen (visualized as tunnels) in the extracellular matrix, where proteolysis of collagen appeared to extend deeply into the extracellular matrix (Figure 2E). This constellation of morphological features, with tunnels extending through damaged basement membrane containing migrating cells, is termed microfracture, because it appears to represent detachments between the epithelium and matrix. Four characteristic features for a microfracture in human amniochorionic membranes are reported. This description is based on the analysis of membranes from term not in labor (Figure 3, A-E) and term labor (Figure 3, F-J) membranes, as well as in vitro cultures in response to cigarette smoke extract (Figure 3, 

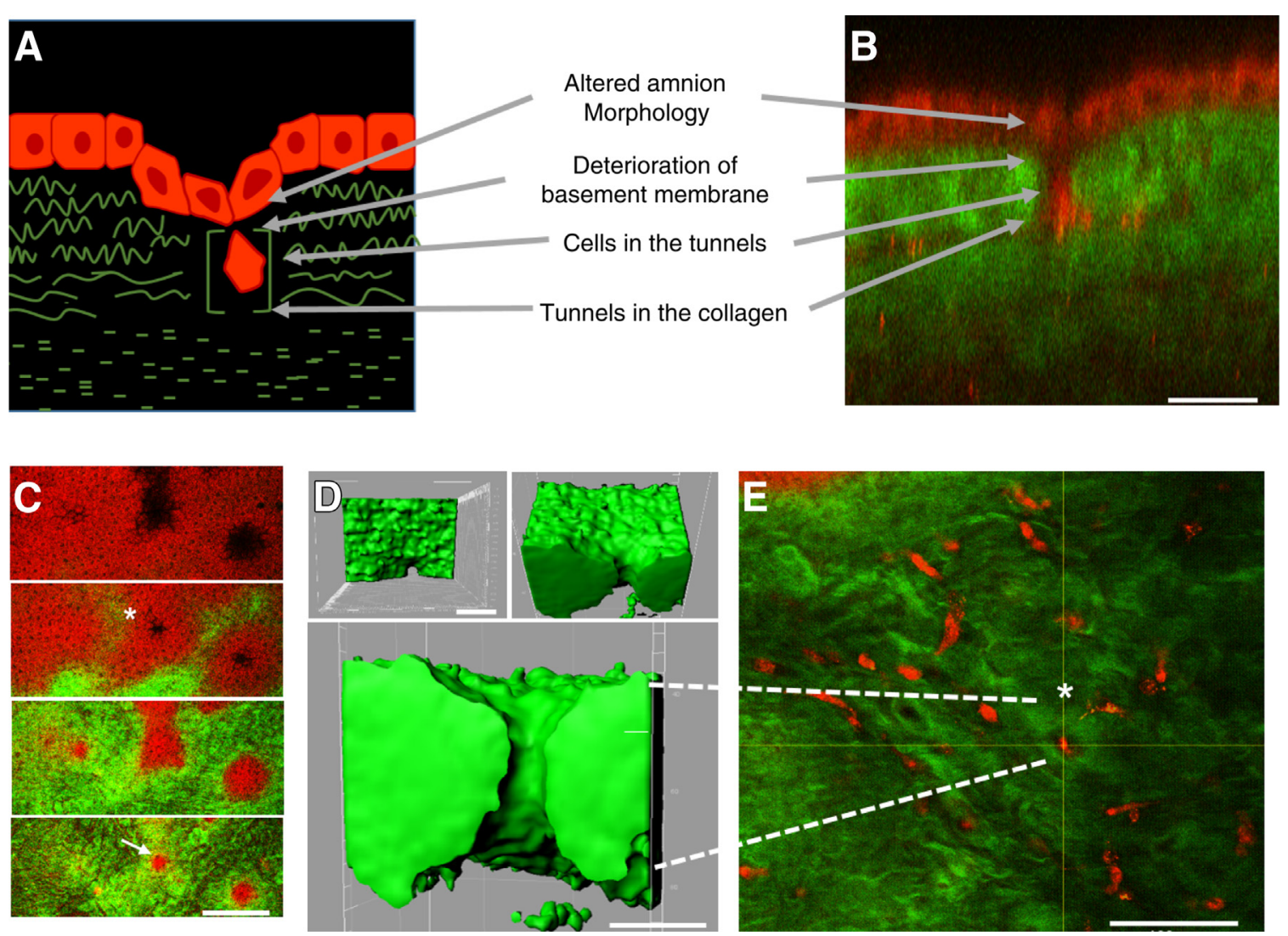

Figure 2 Characteristics of Microfractures. A: Cartoon representation of a microfracture visualized by a degraded tunnel of collagen containing a migrating cell. B: Multiphoton image of microfracture. Cells are red, and collagen, green. C: Multiphoton image of a degraded basement membrane: Representative image of a degraded Type IV rich basement membrane (arrow) around an area of altered amnion morphology (asterisk). D: IMARIS software reconstruction of a degraded tunnel of collagen. E: Multiphoton image of a cell migrating through a degraded collagen tunnel (asterisk). Other stromal cells represented in this image are not in collagen tunnels. Scale bars: $30 \mu \mathrm{m}$ (B); $150 \mu \mathrm{m}$ (C); $10 \mu \mathrm{m}$ (D); $100 \mu \mathrm{m}$ (E).

$\mathrm{K}-\mathrm{O})$ that mimicked the oxidative stress conditions experienced in utero before and during term labor. The main features of microfracture are: i) altered amnion epithelial layer (puckering) or site of epithelial shedding (Figure 3, B, $\mathrm{G}$, and $\mathrm{L}$ ); ii) deterioration and damage of the basement membrane (Figure 3, C, H, and M); iii) tunnels representative of collagen degradation in the extracellular matrix that extend from the basement membrane through the spongy layer (Figure 3, D, I, and N); and iv) the presence of migrating cells in the tunnel (Figure 3, E, J, and O). Identification of microfractures, regardless of condition, suggests that microfractures are a normal constituent of membranes at term. Additionally, membranes from each clinical group also contained microfractures based on the characteristics described above; however, the density, depth, and width of the microfractures differed among the groups. Multiphoton autofluorescence microscopy and second harmonic generation microscopy membrane images $\left(1.50 \times 10^{6} \mu^{3}\right.$ area $)$ analyzed by ImageJ allowed the quantification of differences between term not in labor, term labor, and cigarette smoke extract-treated tissues. Microfracture numbers were similar between the term not in labor and term labor groups (Figure 3P).

\section{In Vitro Induction of Microfractures in Response to 0xidative Stress}

To determine whether oxidative stress can increase microfractures, term not in labor samples were exposed in culture to cigarette smoke extract $(n=5)$. Cigarette smoke extract treatment increased the number of microfractures by 1.62 -fold compared to term not in labor (control) (Figure 3P). IMARIS software was used to create the three-dimensional reconstruction of the collagen matrix region, allowing microfractures to be sliced showing width and depth. Cigarette smoke extract induced deeper $(P<0.001)$ (Figure $3 \mathrm{Q})$ and wider $(P=0.03)$ fractures (Figure 3R) than what was seen in term not in labor controls, suggesting that oxidative stress can cause an increased number of microfractures with increased morphometry. Although the numbers of microfractures are similar in width and depth (range, 10 to $50 \mu \mathrm{mol} / \mathrm{L}$ ) regardless of labor status, there is an increasing trend in each characteristic compared to term not in labor and term labor membranes. We have already reported that the number of senescent cells are higher in term labor compared to term not in labor membranes. ${ }^{18}$ This suggests the possibility that the migratory cells seen in the microfractures at term labor are either shed or senescent cells, which could potentially 

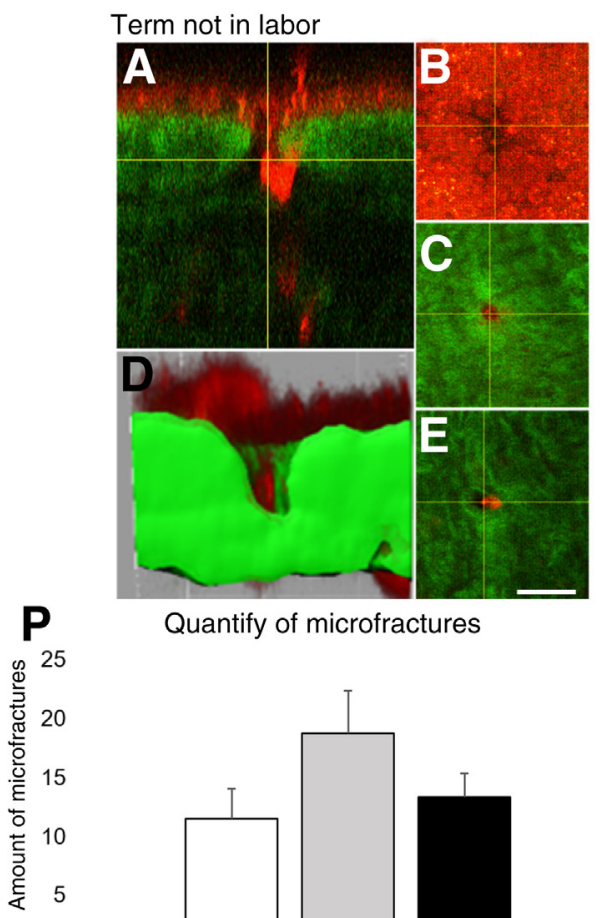

0

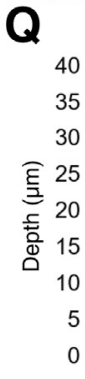

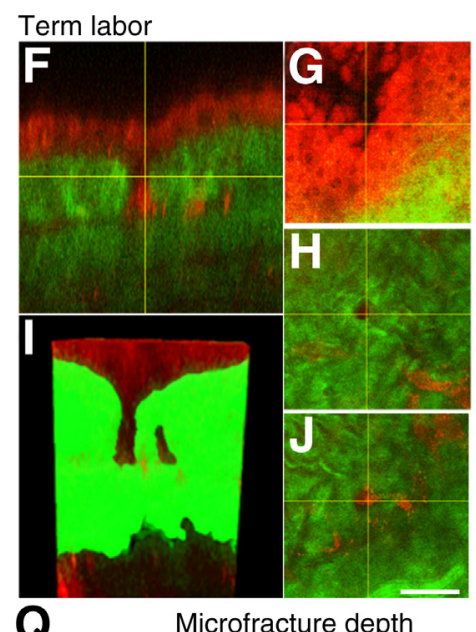

Microfracture depth

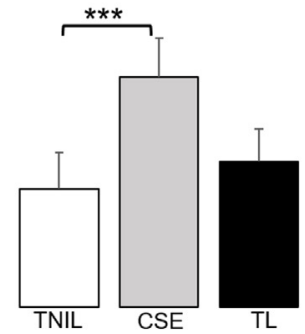

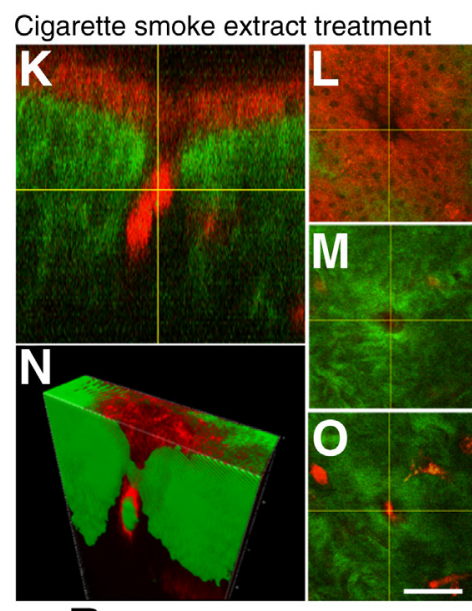

$\mathbf{R}$

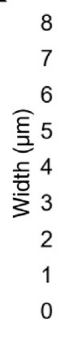

Microfracture width

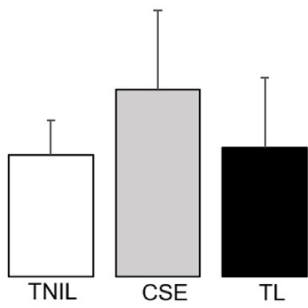

Figure 3 Characteristics and quantification of microfractures in term fetal membrane samples and in membranes exposed to oxidative stress by cigarette smoke extract: $\mathbf{A}-\mathbf{0}$ : Microfractures in term not in labor $(\mathbf{A}-\mathbf{E})$, term labor $(\mathbf{F}-\mathbf{J})$, and cigarette smoke extract-treated tissue in vitro in an organ explant system for 24 hours $(\mathbf{K}-\mathbf{0})$. The yellow cross hairs pinpoint the migrating cell of interest throughout each group of images. $\mathbf{A}, \mathbf{F}$, and $\mathbf{K}: 0$ rthogonal views of a microfracture. B, G, and L: Amnion morphology above the microfracture. C, H, and M: Degraded basement membrane around the migrating cell. D, I, and N: Three-dimensional collagen reconstruction by IMARIS software of a sliced microfracture showing a tunnel of degraded collagen. $\mathbf{E}, \mathbf{J}$, and $\mathbf{0}$ : Degraded collagen tunnel with migratory cell. P-R: Quantitation and morphometric measures of microfractures in term not in labor, term labor, and cigarette smoke extract exposed fetal membranes in vitro. P: Number of microfractures: Multiphoton images add up to a $1.5008 \times 10^{6} \mu \mathrm{m}^{3}$ area that was analyzed for microfracture quantity. Numbers of microfractures were higher after cigarette smoke exposure (CSE) of term not in labor (TNIL) membranes in culture, followed by term labor (TL) membranes. Q: Depth of microfractures: Term labor explants contained deeper microfractures then term not in labor membranes. Cigarette smoke extract treatment produced significantly deeper microfractures than term not in labor controls in vitro or term labor membranes $(P=0.002)$. R: Width of microfractures: Cigarette smoke extract treatment induced wider microfractures compared to term not in labor control cultures $(P=0.0325)$ in vitro. Term labor microfractures were only slightly wider that the term not in labor controls, and they were not significantly different. ${ }^{* *} P<0.001$. Scale bars: $30 \mu \mathrm{m}$ (B, C, E, G, H, J, L, M, 0). All images obtained with a $25 \times$ objective.

traverse through these fractures, though further investigation is required to test this hypothesis.

\section{Microfracture Characteristics of the Chorion and Its Extracellular Region}

The microfractures that are in chorion and its extracellular matrix were also examined. Like amnion membrane, the chorion layer also had microfractures pointing toward the amnion and extending through the reticular layer. Although it is difficult to confirm that the chorion has cell shedding or gap formation (due to its multilayer composition), distorted areas extended toward the matrix region. The reticular layer of the amniochorionic membranes is located on the basal side of the spongy layer and on the apical side of the chorion. Second harmonic generation microscopy microcopy identified loose fibrillar collagen ${ }^{17}$ (Figure 4A) in the reticular layer; here, we add to this description by identifying gaps, potential fractures, in the reticular layer extracellular matrix (Figure 4, B-D), which could be caused by migrating fibroblast or chorion cells. Thus, if microfractures span the gap between the amnion and spongy layer, collagen degradation in the reticular layer could bridge the gap between amnion epithelial cells and the chorion layer, and thus allow the microfractures to span across the membranes.

\section{Microfractures Are More Frequent and Structurally Different in pPROM than Preterm Birth and Preeclampsia}

Increased microfracture density and altered morphometry are responses to oxidative stress and led us to examine membranes from pPROM and preterm birth with no rupture. pPROM has also been termed a "disease of the amniochorionic membranes." 19 pPROM membranes also exhibit increased levels of oxidative stress and collagen degradation. ${ }^{20,21}$ In our study, membranes from gestational age-matched mild preeclampsia were included for two reasons: i) preterm birth following preeclampsia is an indicated delivery; and ii) preeclampsia is not 

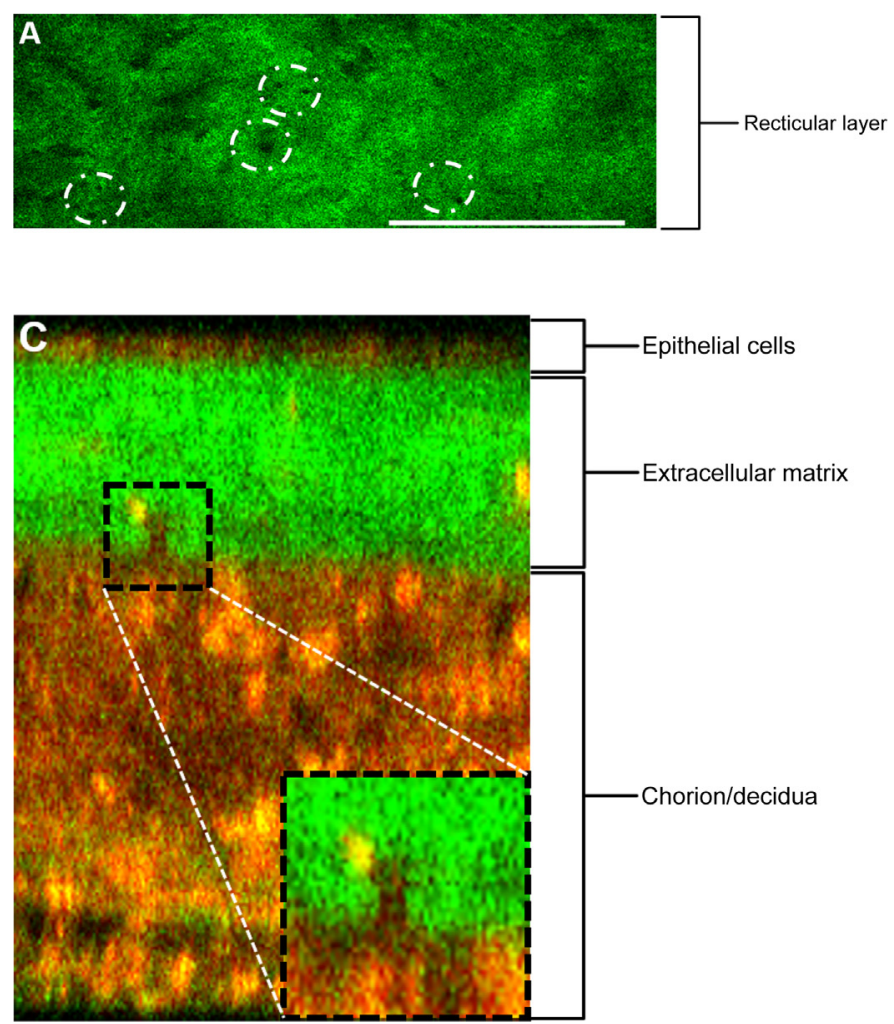

normally associated with membrane dysfunction. Therefore, it may provide a good gestational age-matching control for our pPROM and preterm birth groups, and preeclampsia is often associated with oxidative stress and may provide an oxidative stress-induced effect on membranes that is yet to be reported in this condition. We do acknowledge that membranes in preeclampsia may often show noninflammatory choriodecidual necrosis and/or old retro-membranous blood clots. Membranes from $\mathrm{PPROM}$, preterm birth, and preeclampsia were compared for microfractures and their characteristics (Figure 5, A-L) using multiphoton autofluorescence microscopy and second harmonic generation microscopy as above. Comparison between preterm birth and term birth (regardless of labor status) membranes did not show any difference in the number of microfractures (Figure $5 \mathrm{M}$ ). The number of microfractures in pPROM membranes was (4.6-fold) higher than preterm birth membranes (Figure 5M). Microfractures in pPROM are also deeper (Figure $5 \mathrm{~N}$ ) and wider than in preterm birth membranes (Figure 5O) when three-dimensional reconstructions were formed. To note, the morphometric changes were similar between term labor and preterm birth microfractures, though preterm birth microfractures were significantly wider $(P=0.01)$ than those of term labor.

When membranes from preeclampsia were compared with preterm birth membranes, no significant differences in microfractures (number and dimensions) were seen between the groups. However, comparison between microfractures in preeclampsia and pPROM membranes had similar differences as those seen between pPROM and preterm birth. However, pPROM microfractures are significantly deeper

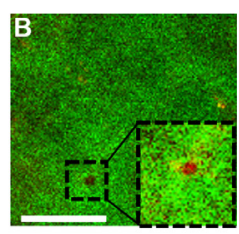

Figure 4 Characterization of the reticular layer. A: Second harmonic generation microscopy image of the reticular layer of amniochorionic membranes. The reticular layer of the chorionic extracellular matrix demonstrating loose collagen and areas of collagen degradation that connect to the chorion (dashed circles). B-D: Multiphoton autofluorescence and second harmonic generation microscopy images of collagen degradation in the reticular layer of the chorion: shown are tunnels of degraded collagen (black box, B), connecting to the chorion layer (white box, D). An overall view of this collagen degradation is depicted in (black box, $C$ ). Insets show a higher magnification of the boxed areas. Scale bars: $150 \mu \mathrm{m}$ (A); $40 \mu \mathrm{m}$ (B). Images in $\mathbf{C}$ and $\mathbf{D}$ were obtained with a $25 \times$ objective.

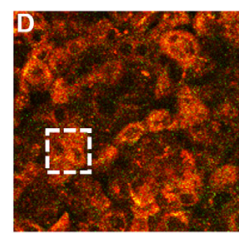

than those seen in preeclampsia $(P=0.04)$. This suggests that the pathologies of preterm birth and preeclampsia are not affecting changes in membrane microfractures. Here, results indicating pPROM had extensive areas of collagen degradation resulting in broader and deeper microfractures are shown. We postulate that these microfractures are areas of insufficient tissue remodeling due to the underlying pathologic condition or premature senescence. Persistent microfractures can act as channels for amniotic fluid leak and inflammatory cell infiltration as often seen in pPROM.

In summary, microfractures appear as tunnels of degraded collagen, possibly vacated by shed or senescent amnion cells. These microfractures could also arise by displacement of chorion leave or trophoblast cells. Collagen degradation, a key characteristic feature of microfractures, extends through the spongy layer of the extracellular matrix, connecting its contents with the dispersed collagen of the reticular layer. The connection created by microfractures between the amnion and chorion may facilitate communication via signals including inflammatory cells and exosomes.

\section{Discussion}

Using a combination of the innovative microscopic techniques of multiphoton autofluorescence and second harmonic generation microscopy, a thorough examination of structural characteristics of normal term amniochorionic membranes (Figure 1) obtained from laboring and nonlaboring women at term and in spontaneous and indicated 
Premature preterm rupture of membranes
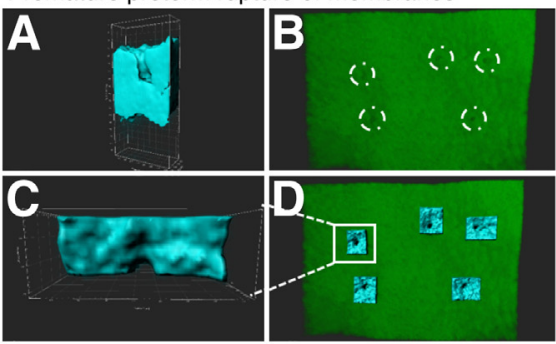

M

M
70

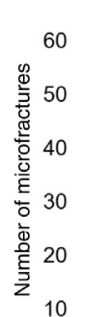

0
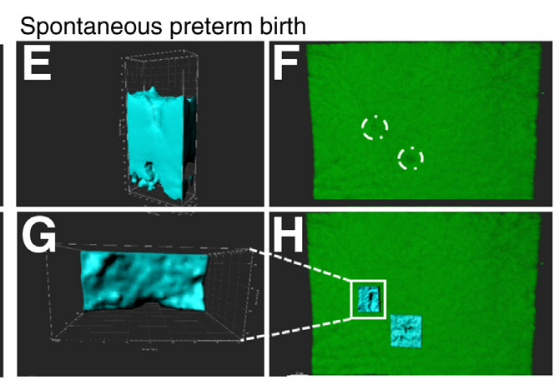

N

$$
30
$$$$
25
$$

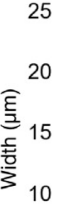

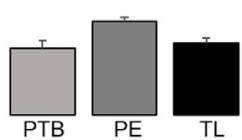

Microfracture depth

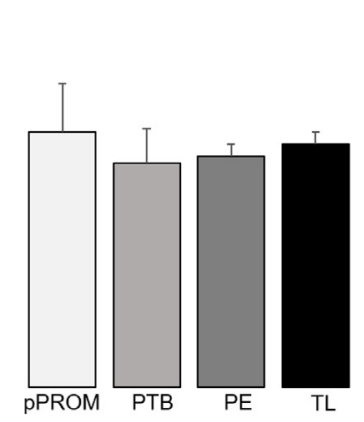

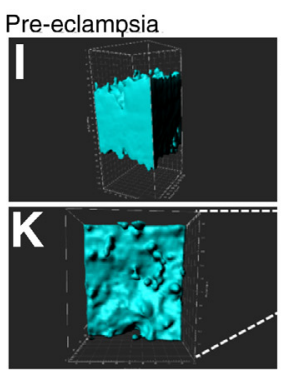
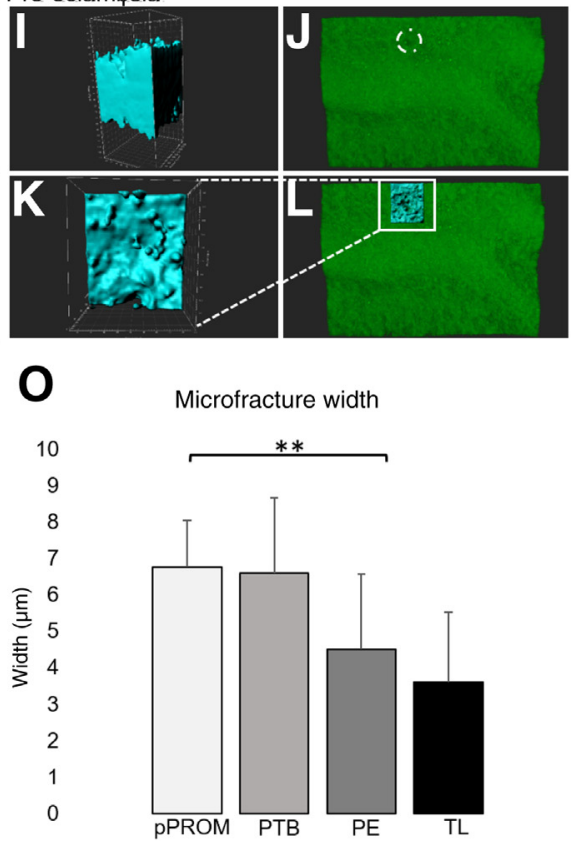

Microfracture width

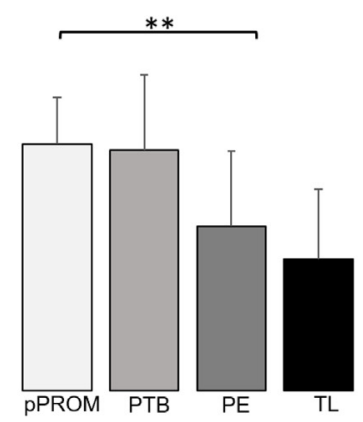

Figure 5 Analysis and quantification of collagen degradation in fetal membranes from preterm birth. Nonlinear optical microscopy analysis of collagen degradation by second harmonic generation microscopy of standardized surface area of fetal membranes from pPROM (A-D), spontaneous preterm birth (PTB) $(\mathbf{E}-\mathbf{H})$, and preeclampsia (PE) (I-L). Surface areas studied [regions of $360 \mu \mathrm{m} \times 260 \mu \mathrm{m}\left(9.4 \times 10^{4} \mu \mathrm{m}^{3}\right)$ ] were similar in the pPROM, spontaneous preterm birth, and preeclampsia samples that were analyzed. A-D: Fetal membranes from pPROM imaged with second harmonic generation microscopy visualizing areas of collagen degradation (dashed circles, B). These areas of collagen degradation, or microfractures, were reconstructed in blue as seen in A, C, and D, highlighting the depth, width, and location of microfractures. pPROM samples had wider and larger numbers of microfractures compared to spontaneous preterm birth and preeclampsia samples. E-H: Fetal membranes from spontaneous preterm birth only contained one true microfracture that was reconstructed and sliced as seen in images E, G, and $\mathbf{H}$. Second harmonic generation microscopy visualized areas of collagen degradation (dashed circles, F). The second blue area in panel $\mathbf{H}$ (not in the white square) may also suggest the beginning of microfracture formation due to the indentation, but not tunnel formation, seen at this location. I-L: Fetal membranes from preeclampsia also showed low numbers and smaller microfractures compared to membranes from pPROM. Second harmonic generation microscopy visualized an area of collagen degradation (dashed circle, $\mathbf{J}$ ). Boxed areas in $\mathbf{D}, \mathbf{H}$, and $\mathbf{L}$ correspond to higher-magnification images in $\mathbf{C}$, $\mathbf{G}$, and $\mathbf{K}$. $\mathbf{M}-\mathbf{0}$ : Quantitation and morphometric measures of microfractures in preterm birth membranes and term labor (TL). M: Number of microfractures: Fetal membranes from pPROM contained a 4.6-fold higher number of microfractures than preterm birth and 3.3-fold higher than preeclampsia samples. N: Depth of microfractures: Microfracture depth did not change much between preterm birth categories or term labor, though PPROM depth is significantly greater than in preterm birth $(P=0.04)$. 0: Width of microfractures: Microfracture widths were not different between pPROM and preterm birth samples $(P=0.005)$; however, compared to term labor, pPROM and preterm birth microfractures were wider. ${ }^{*} P<0.01$. All images obtained with a $25 \times$ objective.

preterm births was performed. In addition, an established amniochorionic membrane explant system under control and oxidative stress conditions was used. The principal finding of this study is the discovery of structural defects in human amniochorionic membranes that we have termed microfractures. These lesions were characterized using the following four morphological criteria: i) the amnion epithelial monolayer showing regions of cell shedding or puckering of amnion cells; ii) localized degradation of the subepithelial basement membrane; iii) fissures or tunnels that extend into the remodeled collagen matrix; and iv) migrating or shed cells that can be identified within the matrix tunnels (Figures 2 and 3). In summary, microfractures represent structural defects that extend into the subepithelial matrix, creating tunnels that can contain satellite amnion cells or other migrating cells. Microfractures can span the thickness of the extracellular matrix, which is around $80 \mu \mathrm{m},{ }^{17}$ and can allow whole amnion cells or their exosomes (microvesicles that are intertissue communication mediators) access to the chorion layer through the loose subepithelial reticulum (Figure 4).

Although collagen and basement membrane degradation are well-reported features in term and pPROM membranes, through the use of advanced three-dimensional multimodal microscopic imaging used in this report, we report for the first time the presence of a characteristic feature termed microfracture that extends from the amnion through the extracellular matrix and that reaches the chorion layer with altered cellular and matrix morphology. Microfractures are likely developed due to a structural alteration created by biochemical- and cellular-level changes (Figure 5). These findings were not possible previously, because traditional histologic and microscopic approaches such as immunohistochemistry or confocal microscopy are not adequately sensitive, either lacking the ability for volumetric threedimensional assessment or lacking the specific contrast from collagen provided by second harmonic generation microscopy. 
Microfractures may develop when amniochorionic membranes expand and grow to support fetal development. Membrane growth and extension involve amnion cell shedding and gap formation, leading to microfracture formation. Our ongoing studies and recently reported findings suggest that distorted or displaced amnion epithelial cells can be recycled or transitioned to become mesenchymal cells (epithelial to mesenchymal transition), ${ }^{22}$ and this transition involves localized inflammation that involves collagen metabolism. This will lead to microfracture resealing, a mechanism to remodel membranes. Microfractures are likely areas where active remodeling takes place during gestation, and their development and resealing are essential to main membrane integrity. Data from mild preeclampsia (a condition where amniochorionic membrane dysfunctions are not reported) may support this concept, where a small number of microfractures along with collagen rearrangement was seen (Figure 5). Increased quantity of microfractures and pathologic conditions that will fail to reseal may predispose membranes to rupture, resulting in an adverse pregnancy.

An increase in the number of microfractures and their dimensions was visible after oxidative stress induction in vitro and in membranes from pPROM (Figure 5), a condition associated with increased oxidative stress. ${ }^{23}$ Development of microfractures in response to oxidative stress-inducing risk factors (infection, poor nutrition, high body mass index, behavioral risks, etc) can be a predisposing factor for pPROM because microfractures can provide channels for amniotic fluid leak and inflammatory cell migration. We also speculate that microfractures can act as channels of communication by fetal cell-derived exosomes that also carry inflammatory signals at term or in response to oxidative stress. ${ }^{24}$

Our prior reports showed several biochemical and histologic senescence and oxidative stress-associated markers that were similar in term labor and pPROM membranes. ${ }^{9}$ However, microfractures are not similar in numbers or morphometry between term labor and pPROM membranes. Although senescence is a factor associated with both conditions, ${ }^{9}$ microfractures are likely independent of senescence-associated changes. As mentioned above, microfractures are likely sites of membrane remodeling, and pPROM is a condition associated with tissue destruction due to remodeling failures. The lack of differences in the number of microfractures between two term groups (term not in labor and term labor) suggests pluripotency of cells in the membrane. These cells retain the ability to reseal microfractures even at term, whereas pPROM membranes have a diminished capacity to reseal.

Due to limited sample sizes in each of the categories, the specific role of histologic chorioamionitis or microbial invasion in the spontaneous preterm birth or PPROM groups were not examined. Although this was not one of the objectives of this study, some of these factors may affect microfracture development, resealing, and/or its persistence leading to rupture. These pathologic factors along with other oxidative stress-inducing risk factors and their impact on microfracture formation/tissue remodeling or destruction are the focus of our ongoing studies. Another limitation of this study comes in sample preparation due to tissue fixation preventing directionality studies. Future studies will use live tissues to better document this remodeling process.

In summary, the clinical significance of microfractures is highlighted. We postulate that persistence of microfractures or failure to repair them might lead to pockets of inflammation in the membranes. Our ongoing studies have shown matrix metalloproteinase 9 immunostaining, suggesting inflammatory and matrix degradation-related changes. Microfractures can function as channels of amniotic fluid leak, and failure to reseal microfractures can be considered as a predisposing factor for membrane rupture. Several pioneering studies have reported extracellular matrix remodeling and collagen metabolism in amniochorionic membranes and their overwhelming activities in response to infection and other risk factors causing membrane rupture. This report provides more evidence to suggest that pPROM is a disease of the fetal membranes. Further studies centered on microfractures could provide valuable insights into their development and other functional roles, as well as how they contribute to membrane dysfunctions.

\section{Acknowledgments}

We thank Dr. Hui-Juan Zhang for critical review of the manuscript and valuable suggestions and Jayhsil Trivedi for help collecting clinical samples.

\section{References}

1. Menon R: Human fetal membranes at term: dead tissue or signalers of parturition? Placenta 2016, 44:1-5

2. Vadillo-Ortega F, Hernandez A, Gonzalez-Avila G, Bermejo L, Iwata K, Strauss JF III: Increased matrix metalloproteinase activity and reduced tissue inhibitor of metalloproteinases-1 levels in amniotic fluids from pregnancies complicated by premature rupture of membranes. Am J Obstet Gynecol 1996, 174:1371-1376

3. Fortunato SJ, Menon R, Lombardi SJ: MMP/TIMP imbalance in amniotic fluid during PROM: an indirect support for endogenous pathway to membrane rupture. J Perinat Med 1999, 27:362-368

4. Athayde N, Romero R, Gomez R, Maymon E, Pacora P, Mazor M, Yoon BH, Fortunato S, Menon R, Ghezzi F, Edwin SS: Matrix metalloproteinases-9 in preterm and term human parturition. J Matern Fetal Med 1999, 8:213-219

5. Menon R, Behnia F, Polettini J, Saade GR, Campisi J, Velarde M: Placental membrane aging and HMGB1 signaling associated with human parturition. Aging (Albany NY) 2016, 8:216-230

6. Menon R, Bonney EA, Condon J, Mesiano S, Taylor RN: Novel concepts on pregnancy clocks and alarms: redundancy and synergy in human parturition. Hum Reprod Update 2016, 22:535-560

7. Bryant-Greenwood GD: The extracellular matrix of the human fetal membranes: structure and function. Placenta 1998, 19:1-11

8. Malak TM, Bell SC: Structural characteristics of term human fetal membranes: a novel zone of extreme morphological alteration within the rupture site. Br J Obstet Gynaecol 1994, 101:375-386 
9. Menon R, Boldogh I, Hawkins HK, Woodson M, Polettini J, Syed TA, Fortunato SJ, Saade GR, Papaconstantinou J, Taylor RN: Histological evidence of oxidative stress and premature senescence in preterm premature rupture of the human fetal membranes recapitulated in vitro. Am J Pathol 2014, 184:1740-1751

10. Pal R, Shilagard $T$, Yang J, Villarreal $P$, Brown $T$, Qiu S, McCammon S, Resto V, Vargas G: Remodeling of the epithelialconnective tissue interface in oral epithelial dysplasia as visualized by noninvasive 3D imaging. Cancer Res 2016, 76:4637-4647

11. Mukherjee S, Wysock JS, Ng CK, Akhtar M, Perner S, Lee MM, Rubin MA, Maxfield FR, Webb WW, Scherr DS: Human bladder cancer diagnosis using Multiphoton microscopy. Proc SPIE Int Soc Opt Eng 2009, 7161:nihpa96839

12. Chowdhury B, David AL, Thrasivoulou C, Becker DL, Bader DL, Chowdhury TT: Tensile strain increased COX-2 expression and PGE2 release leading to weakening of the human amniotic membrane. Placenta 2014, 35:1057-1064

13. Jabareen M, Mallik AS, Bilic G, Zisch AH, Mazza E: Relation between mechanical properties and microstructure of human fetal membranes: an attempt towards a quantitative analysis. Eur J Obstet Gynecol Reprod Biol 2009, 144 Suppl 1:S134-S141

14. Mauri A, Ehret AE, Perrini M, Maake C, Ochsenbein-Kolble N, Ehrbar M, Oyen ML, Mazza E: Deformation mechanisms of human amnion: quantitative studies based on second harmonic generation microscopy. J Biomech 2015, 48:1606-1613

15. Mauri A, Perrini M, Mateos JM, Maake C, Ochsenbein-Koelble N, Zimmermann R, Ehrbar M, Mazza E: Second harmonic generation microscopy of fetal membranes under deformation: normal and altered morphology. Placenta 2013, 34:1020-1026

16. Perrini M, Mauri A, Ehret AE, Ochsenbein-Kolble N, Zimmermann R, Ehrbar M, Mazza E: Mechanical and microstructural investigation of the cyclic behavior of human amnion. J Biomech Eng 2015, 137: 061010

17. Richardson L, Vargas G, Brown T, Ochoa L, Trivedi J, Kacerovský M, Lappas M, Menon R: Redefining 3Dimensional placental membrane microarchitecture using multiphoton microscopy and optical clearing. Placenta 2017, 53:66-75

18. Behnia F, Taylor BD, Woodson M, Kacerovsky M, Hawkins H, Fortunato SJ, Saade GR, Menon R: Chorioamniotic membrane senescence: a signal for parturition. Am J Obstet Gynecol 2015:213: $\mathrm{e} 1-\mathrm{e} 16$

19. Murtha AP, Menon R: Regulation of fetal membrane inflammation: a critical step in reducing adverse pregnancy outcome. Am J Obstet Gynecol 2015, 213:447-448

20. Parry S, Strauss JF III: Premature rupture of the fetal membranes. N Engl J Med 1998, 338:663-670

21. Arechavaleta-Velasco F, Ogando D, Parry S, Vadillo-Ortega F: Production of matrix metalloproteinase-9 in lipopolysaccharide-stimulated human amnion occurs through an autocrine and paracrine proinflammatory cytokine-dependent system. Biol Reprod 2002, 67: $1952-1958$

22. Janzen C, Sen S, Lei MY, Gagliardi de Assumpcao M, Challis J, Chaudhuri G: The role of epithelial to mesenchymal transition in human amniotic membrane rupture. J Clin Endocrinol Metab 2017, 102:1261-1269

23. Dutta EH, Behnia F, Boldogh I, Saade GR, Taylor BD, Kacerovsky M, Menon R: Oxidative stress damage-associated molecular signaling pathways differentiate spontaneous preterm birth and preterm premature rupture of the membranes. Mol Hum Reprod 2016, 22:143-157

24. Sheller-Miller S, Lei J, Saade G, Salomon C, Burd I, Menon R: Fetomaternal trafficking of exosomes in murine pregnancy models. Front Pharmacol 2016, 7:432 